\title{
Téoros
}

Revue de recherche en tourisme

\section{La reproduction touristique des patrimoines et le développement touristique dans les fêtes populaires en Mauricie}

\author{
Éric Schwimmer et Michel Chartier
}

Volume 5, numéro 3, novembre 1986

URI : https://id.erudit.org/iderudit/1080543ar

DOI : https://doi.org/10.7202/1080543ar

Aller au sommaire du numéro

Éditeur(s)

Université du Québec à Montréal

ISSN

0712-8657 (imprimé)

1923-2705 (numérique)

Découvrir la revue

Citer cet article

Schwimmer, É. \& Chartier, M. (1986). La reproduction touristique des patrimoines et le développement touristique dans les fêtes populaires en Mauricie. Téoros, 5(3), 41-42. https://doi.org/10.7202/1080543ar d'utilisation que vous pouvez consulter en ligne. 


\title{
La reproduction touristique des patrimoines et le développement touristique dans les fêtes populaires en Mauricie
}

\author{
par Eric Schwimmer et Michel Chartier"
}

\section{Le projet}

Nous rendons compte brièvement ici, d'une recherche qui démarrera bientot et qui porte, de manière gếnérale, sur la fete populaire au Québec. Nous avons sélectionné pour l'instant, un échantillon de neuf fêtes, localisées en Mauricie, à partír d'informations tirées du "Guide Touristique" "1l! de la région du Coeur-du-Québec. L'équipe de recherche est composée d'enseignants et d'étudiants du Département d'Anthropologie de l'Université Laval à Québec. L'objet de cet exposé sommaire, est de sensibiliser le monde du tourisme a ce que nous voulons faire et d'instaurer si possible un dialogue.

Car si notre recherche sur la fête populaire doit avoir des retombes pratiques, cellesci se situeront sans doute dans le champ du développement touristique. Expliquons d'abord le projet et posons ensuite quelques questions sur sa pertinence au tourisme.

\section{La fête}

La fête est une activitité périodique, paisible et collective qui rétablit l'equilibre social en permettant l'expression de certaines tendances refoulées dans la vie quotidienne. Même si plusieurs théoriciens ont regardé la fête comme un événement cyclique et répétitif, nous croyons qu'elle fait partie aussi du temps irréversible, c'est-âdire de l'Histoire. Il semble probable que ce rôle historique très évident dans le cas des fêtes de la Saint-Jean-Baptiste, sera tout aussi important dans les festivités mauriciennes que nous voulons étudier.

Conme microcosme de l'imaginaire du Québécois contemporain, la fête exprime d'abord le symbol isme du patrimoine, et le désir de s'intégrer à une image du patrimoine, mais cette image est en transformation constante, si bien que le patrimoine ne se reconnait dans la fete que sous des formes sans cesse renouvelées, crée par la conscience contemporaine. L'imaginaire de la culture populaire ne se limite pourtant jamais à ces constructions cognitives plus

"MM. Eric Schwimmer et Michel Chartier sont professour au Département d'Anthropologie de la Faculté de scionces sociales de l'Universite Laval. ou moins savantes, mais, comme on sait, par la joie, par la bouffe, la bière, le vêtement festif, par les performances de toute sorte, par un comportement libre et dóchaîné, parfois violent et saccageur, et surtout par la licence de faire ce qu'on n'oserait jamais faire dans la vie quotidienne. L'analyste peut y reconnaître aussi, une façon différente, voir même invertie, d'aménager l'espace et la présentation de certains objets symboliques exprimant un lien d'identité culturelle moins actualise dans la vie culturelle. Dans ces circonstances, comment s'exprime-t-on exactement? Voilà la question à laquelle notre projet a l'intention de répondre.

\section{La méthode}

Du point de vue méthodologique, ce projet utilisera une approche en grande partie sémiotique. On va donc analyser certains codes comme celui du vêtement, de la bouffe, de la bière. Mais il y a aussi quelques codes complexes comme celui de l'argent et du gaspillage; le gaspillage et la prudence quasi marchande s'y cốtoyant dans une relation dialectique. Il y a aussi le code religieux, car la fête est regardée par l'Église avec une grande tolérance, avec la reconnaissance qu'elle est inévitable et qu'elle peut jouer un rôle positif, mêtme si elle donne lieu inévitablement à certaines transgressions. Cependant, la fête est souvent loin de la religion officielle, sans jamais devenir athéiste: elle fait partie de ce qu'on appelle la religion populaire. Il semble probable que notre enquête démontrera que la fête populaire québécoise est en véritế profondément religieuse. Finalement, l'étude ne serait pas complète si elle ne regardait pas le code sexuel, et si elle n'analyserait pas le discours des fêtards, et les themes sur lesquels il porte.

\section{Le tourisme}

Dans cet ordre général d'idées, le tourisme est un aspect particulier de la fette. Car il a le même caractère périodique, paisible et souvent collectif. Comme le fêtard, le touriste cherche une certaine image de soi dans lạ détente; il veut être lui-méme mais dans le sens festif. Il aime donc souvent assister aux fêtes; celles-ci constituent une attraction touristique certaine. Cela pose des questions pratiques d'aménagement, car la capitalisation touristique exige que les equipements soient utilisés pour une saison plus longue que la durée des fêtes. Les fêtes n'attirent pas le touriste traditionnel à moins qu'elles ne fassent partie d'un complexe plus vaste d'attraction permanentes.

Notre recherche analysera les fêtes populaires comme une extension de la grande fête familiale. Nous voulons tablir la provenance du public qui y assiste et surtout quelle place les membres de la parenté, des amis, et d'autres personnes liées au festival par des rapports et des intérêts particuliers occupe dans ce public.

Nous voulons distinguer ce tourisme associé à la participataion à une fête du tourisme plus généralement pratiqué (visite de site. etc.); celui-ci propose habituellement des attractions sans liens directs avec les valeurs sociales et les idéologies particulières d'un lieu visité. Cette distinction estelle valable? Comment les théories du tourisme l'aborde-t-elle? En fait, tout touriste quelqu'il soit, n'est-il pas exposé aux valeurs du milieu qu'il visite d'une façon ou d'une autre? Nous aurons à interroger ces touristes ordinaires autant que ceux qui se déplacent pour les fêtes.

\section{Les données de base}

Ajoutons maintenant, quelques précisions concernant l'échéancier du projet et la délimitation de l'espace social où se déroulera les fêtes que nous avons sélectionnées; ensuite nous examinerons les donnés de base que nous possédons déjà, en regard de la problématique exposée précédemment.

L'enquête extensive devra en principe commencer le ler juin 1987 et se terminer le 31 mai 1989, Or, si pendant ces deux années nous obtenons des résultats pratiques, qui promettent d'avancer notre connaissance générale du phénomène de la fête populaire au Québec, il est possible que le projet s'étende au-delà de cette date. Une enquête préliminaire est déjà en cours cet été. Elle permettra entre autre, de reviser la liste des fêtes, si cela s'avérait necessaire. 
Nous avons privilégié certains territoires à I'exclusion d'autres, en fonction de frontières sociales bien détinies, Excluant tous les secteurs touristiques de la rive sud du Fleuve Saint-Laurent, I'espace à étudier correspond globalement à trois secteurs touristiques de la région mauricienne: $\mathrm{A}$ Trois-Rivières, B- Le chemin du Roy, et C- La vallée du Saint-Maurice. Ce territoire est délimité au sud par la rive nord du Fleuve Saint-Laurent, au nord par la ville de La Tuque, et s'êtend d'est en ouest entre les $72^{\circ}$ et $73^{\circ}$ de longitude.

\section{La Mauricie comme espace géographique et touristique}

Espace géographique et touristique la Mauricie doit aussi etre vue dans son entite sociale, et dans les rapports sociaux qu'elle engendre. Ainsi, avant de proposer tout projet d'aménagement, nous croyons qu'il est utile d'analyser le phénomène des fêtes populaires, comme pratique touristique a trois points de vue: 1 . de la circulation des réseaux sociaux internes à la rếgion qui débordent vers l'extérieur (et vice versa); 2 . de la reproduction des patrimoines familiaux; et 3. de l'échange des biens matếriels et immatériels. Ce dernier point de vue est essentiel à notre démarche, car beaucoup de fêtes ne sont explicables que dans le contexte de la reproduction (economique, politique et religieuse) des groupes.

Bref, si on veut definir la Mauricie comme un espace touristique régional, économiquement exploitable de ce point de vue, il ne faut pas négliger avant tout de la considérer comme un espace social en soi, où les membres et anciens membres (incluant sur le plan symbolique les ancêtres-morts) de chaque communauté locale et parentale, envahissent cet espace pendant ces périodes festives, circulent à l'intérieur de leurs frontières pour echanger des visites à leurs parents, amis et voisins, ou les débordent pour justement fuir la fête. Par ailleurs, si le contexte historique des fêtes populaires en Mauricie favorise de grands rassemblements et augmenta le nombre de ces ếvếnemenis depuis vingt ans, on peut penser aussi, que c'est gräce ă l'extension des réseaux de relations inter-localités et interfamiliales, en vue de la reproduction des groupes qui la composent, que de telles réunions sont possibles.

Afin de rétablir les faits concernant ces grandes concentrations, nous allons mettre l'accent sur l'étude des réseaux sociaux spécifiques qui rayonnent à partir d'un point central localisé à Sainte-Tite. En s'interrogeant sur la elientèle touristique qui fréquente cette ville, pendant la période du Festival Western, des rapports qu'elle entretient avec cette population locale, nous nous demanderons alors si les habitants de Saint-Tite echangent hl leur tour des visites et assistent à d'autres fêtes ailleurs, et oc en fonction des réseaux que nous aurons découvert. Nous vérifierons, ensuite ce modèle des réseaux en l'appliquant à chacune des localités à l'étude.

Sur le plan économique, l'industrie touristique, d'après I'A.T.R. du Coeur-duQuébec: "tient un rôle secondaire, de support et d'appui" par rapport aux autres activités economiques de la région. (2) Mais admettons quand meme que l'argent investi depuis une vingtaine d'années, en Mauricie, dans l'aménagement de la nature comme site de villégiature (ex: le Parc National de la Mauricie, la Réserve Faunique du Saint-Maurice, les ZECS, etc.). de loisirs, de chasse et de pëche a de toute evidence aidé cortains groupes à se refaire, à court terme, un capital politique et financier.

\section{Fêtes et dimension économique}

Situées à proximité de ces sites, les localités où se déroulent les fëtes profitent des touristes qui arrêttent au passage. La féte populaire fait done partie du circuit touristique. Dépendantes en grande partie des politiques de subventions gouvernementales, des nombreux commanditaires (brasseries, caisses populaires, plusieurs commerces locaux, etc.), les fètes populaires s'insèrent dans une mème visée économique et politique: attirer de plus en plus de touristes pour promouvoir l'exploitation de certaines ressources naturelles (I'orignal, le poulamon), vendre certains produits locaux (le cuir, le bois, les canots, le blé d'Inde, l'artisanat, etc.), les produits nationaux et d'importation, et aussi pour louer toute sorte d'equipement. En fait, derrière chaque promotion publicitaire se cache une certaine idéologie mercantile qui incite les touristes à dépenser pour mieux fêter. Ce qui nous intéresse dans cette dernière question, c'est l'étude des représentations que le touriste-fetard-consommateur accorde à la valeur de l'argent en ces périodes de festivites; et d'autre part, l'aspect positif de la fête qui anéantit ou neutralise toute critique négative à l'égard de ceux qui font des profits avec l'argent des fetards. Ceux-ci profitent aussi, dans une certaine mesure, de ceux-là car ils font partie de la fête. Par exemple, les compagnies de bières investissent beaucoup d'argent dans ces festivités et à voir les centaines de millier de gallons de bières qui se boivent lors de ces événements, on constate une certaine relation de complicité entre le brasseur et le buveur. Ce type de complicité vaut aussi pour les autres vendeurs de fétes: boutiquiers d'accessoires festifs, d'artisanats, de gadgets variés; les restaurants de bouffe träditionnelle, de fast food, et la bouffe qu'on trouve seulement dans ces occasions.

Finalement, nous essaierons de voir dans quelle mesure la Mauricie se donne des airs de fêtte à l'américaine. Le cas apparcnt du Festival Western de Saint-Tite est la première cible visce par les critiques. Cepen- dant, du point de vue de l'analyse, nous pensons que le décor est une forme de simulacre: il peut être américain, russe, papou, voir même québécois décrivant un patrimoine passéiste, mais l'expression festive profonde, demeure authentique en son fond populaire. Cette résistance se traduit surtout par des éléments d'une tradition fortement empreinte de la religiosité primitive. Ce qui se vit sur la place publique déborde largement les cadres imposés de l'extérieur ct même ceux proposés par l'organisation officielle locale. Il ne faut pas nier toutefois certaines transformations historiques qui sont survenus aux cours des siècles.

$f$

(1) Ce document est une brochure publiée par le Ministẻre du Tourisme en collaboration avec I'Association Touristique lou A.T.R.) du "Coeur-du-Quebesc", 2e ediction, 1986.

(2) CAZELAIS, Normand: "Rible et action des associations fowristiques régionales" in Teroros, Vol. 5, no. 1, mars 1996, p. 31

Les associations

(Suite de la page 35)

Cette refonte des programmes d'aide, que le gouvernement tarde à entreprendre, devrait, à notre avis, être l'occasion d'examiner également les alternatives, ou plutôt les compléments, à l'aide gouvernementale.

Ces alternatives ou compléments pourraient, par exemple, viser à mobiliser une épargne qui n"emprunterait pas les circuits traditionnels de placement. Les experriences européennes comme celle, récente, du titre associatif, nous apparaissent intéressantes à examiner.

Chose certaine, l'Etat aura toujours une responsabilitế à assumer dans le secteur du tourisme associatif si l'on veut que l'aceessibilite aux vacanos et au touristhe soit une réalité au Québec. Les formes d'aide employées jusqu'ici n'ont pas contribué, à notre avis, à développer un secteur hautement performant au plan de la gestion, mème s'il peut se comparer avantageusement à d'autres secteurs touristiques traditionnels. Une refonte des programmes d'aide devra avoir comme préoceupation cette nécessaire performance.

Les moyens qu'il faudra mettre de l'avant pour assurer la consolidation et le développement du secteur associatif devront également tenir compte d'un nécessaire maintien de sa spécificité. Partic intégrante de l'économie touristique du Québec, le secteur associatif doit demeurer le principal animateur d'un tourisme accessible offrant des prestations de qualité à des prix abordables. Qui d'autres pourraient jouer ce rôle? $f$

\section{Notes}

(1) II s'agit ici d'hypotheses de travail. 